[Supporting Information for:]

\title{
Enantioselective MSPV Reduction of Ketimines using 2-Propanol and (BINOL)AI ${ }^{\text {III }}$
}

\author{
Christopher R. Graves, Karl A. Scheidt,* and SonBinh T. Nguyen* \\ Department of Chemistry and the Institute for Environmental Catalysis, Northwestern University, 2145 Sheridan Road, \\ Evanston, Illinois 60208-3113, USA
}

General Procedures, Materials, and Instrumentations. All air- or water-sensitive reactions were carried out under nitrogen either in a Vacuum Atmospheres Company drybox or using standard Schlenk-line techniques. Triethylamine (Fisher Scientific) was dried over $\mathrm{CaH}_{2}$ and vacuum-transferred into a Strauss flask. Toluene (Fisher Scientific) was dried over alumina and Q5 catalyst via the Dow-Grubbs solvent system ${ }^{1}$ installed by Glass Contours (Laguna Beach, CA). 2Propanol (Fisher Scientific) and 2-propan-2- $d$-ol (Aldrich Chemicals) were distilled over $\mathrm{Mg}\left(\mathrm{O}^{i} \operatorname{Pr}\right)_{2}$ and $\operatorname{Mg}\left(\mathrm{O}^{i} \operatorname{Pr}-2 d\right)_{2}$, respectively. $\mathrm{C}_{6} \mathrm{D}_{6}$ (Cambridge Isotope Laboratories) was dried over sodium and benzophenone ketyl and vacuumtransferred into a Strauss flask. All solvents and reagents were saturated with nitrogen and stored in Strauss flasks prior to use. $\mathrm{CDCl}_{3}$ (Cambridge Isotope Laboratories), enantiomerically pure samples of 2,2'-dihydroxy-1,1'-binapthyl (BINOL) (Sumikin Chemical Company, now Air Water Chemical, Inc. http://www.aw-chem.co.jp/e/index.html), and all other reagents (Aldrich Chemical Company) were purchased from commercial sources and used without further purification, unless otherwise noted.

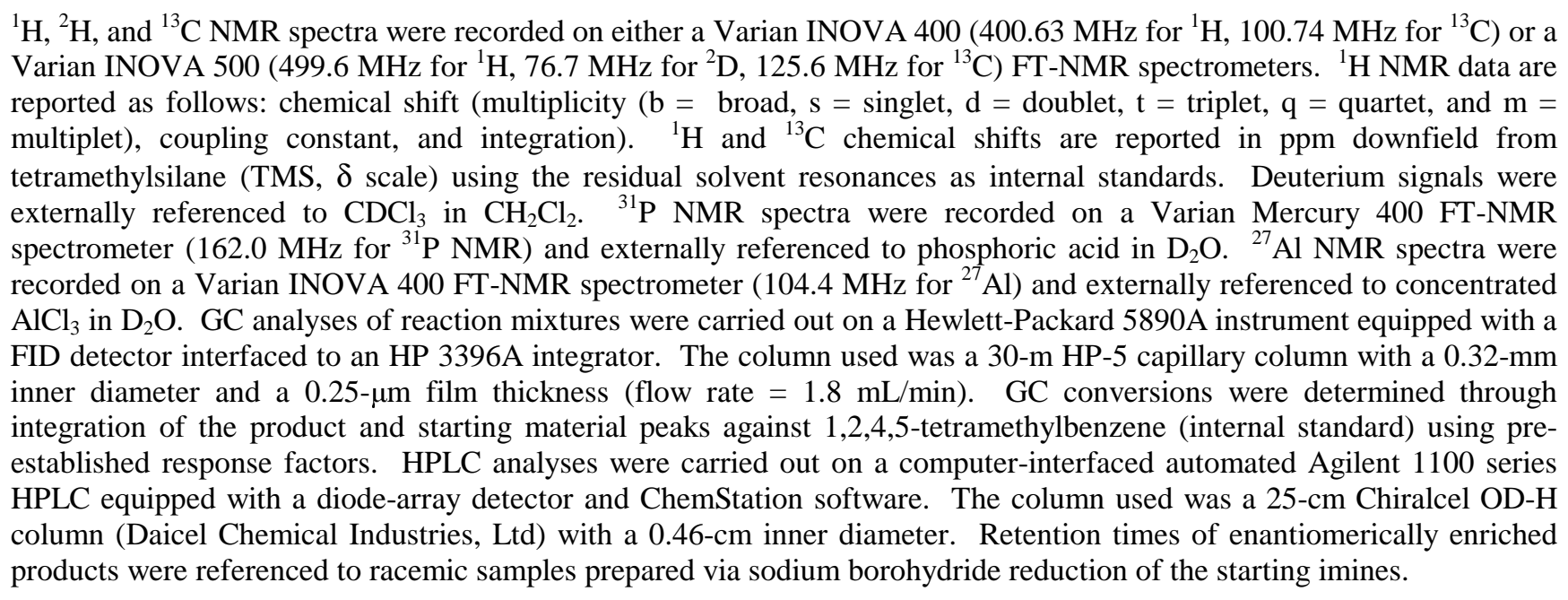

General Procedure for the synthesis of imine starting materials. Imines 1-10 were synthesized following modified literature procedures. ${ }^{2}$ Under bench-top conditions and in a $100-\mathrm{mL}$ round-bottom flask equipped with a magnetic stir bar, the appropriate oxime $(7.4 \mathrm{mmol})$ was combined with anhydrous toluene $(10 \mathrm{~mL})$ and anhydrous triethylamine $(0.75 \mathrm{~g}, 7.4$ mmol). The resulting mixture was stirred at room temperature and $\mathrm{PPh}_{2} \mathrm{Cl}(1.6 \mathrm{~g}, 7.4 \mathrm{mmol})$ in anhydrous toluene $(5 \mathrm{~mL})$ was added drop-wise via addition funnel ( $\sim 0.5 \mathrm{~h}$ addition time). Upon completion, the reaction was stirred for an additional hour at room temperature after which precipitate was filtered from the reaction using a Büchner funnel. Solvents were removed from the filtrate using a rotary evaporator, leaving a pale yellow crude reaction product. Subsequent purification via column chromatography on silica gel $(15 \mathrm{~cm} \times 5 \mathrm{~cm})$ with acetone $/ \mathrm{CH}_{2} \mathrm{Cl}_{2}$ eluants (10/90-50/50) afforded the pure products as white to off-white solids except 10, which was a pale yellow oil (yield $=35-60 \%$ ).

General procedure for optimization of the MSPV reduction of 1 . In the drybox, an 8-mL vial equipped with a magnetic stir bar was charged with $(S)$-BINOL $(0.16-0.19 \mathrm{mmol})$. Anhydrous toluene $(1-3 \mathrm{~mL})$ was added followed by $\mathrm{AlMe}_{3}$ $(0.16-0.19 \mathrm{mmol})$ and the vial was capped with a Teflon-lined silicone septa. The resulting cloudy mixture was stirred for $0.5 \mathrm{~h}$ when 2-propanol was added. The ensuing homogeneous reaction was allowed to stir at room temperature for an additional $0.5 \mathrm{~h}$, taken out of the drybox, and then heated to $60{ }^{\circ} \mathrm{C}$ in a Thermolyne Type 17600 Dri-Bath block heater equipped with an aluminum heating block containing a $4 \times 3$ bank of [20 mm diameter $\times 50 \mathrm{~mm}$ depth] wells. A solution of the imine $(0.16 \mathrm{mmol})$ in toluene $(1 \mathrm{~mL})$ was then added via a gas-tight syringe and the resulting yellow solution was stirred for an additional $20 \mathrm{~h}$ at $60{ }^{\circ} \mathrm{C}$. A $100-\mu \mathrm{L}$ fraction was taken via gas-tight syringe, diluted in $\mathrm{CH}_{2} \mathrm{Cl}_{2}(3 \mathrm{~mL})$, and extracted with water $(3 \times 5 \mathrm{~mL})$. The organic layer was collected, dried over anhydrous $\mathrm{Na}_{2} \mathrm{SO}_{4}$, and directly analyzed via $\mathrm{GC}$ and HPLC. GC temperature program: Initial temp $=50{ }^{\circ} \mathrm{C}$, initial time $=5 \mathrm{~min}$; ramp rate $=10{ }^{\circ} \mathrm{C} / \mathrm{min}$, final temp $=$ 
$250{ }^{\circ} \mathrm{C}$, final time $=20 \mathrm{~min}$ (imine retention time $=31.4 \mathrm{~min}$; amine retention time $=30.6 \mathrm{~min} ;$ BINOL retention time $=$ $28.4 \mathrm{~min})$. HPLC conditions are listed below.

General procedure for the MSPV reduction of imines. In the drybox, an 8-mL vial equipped with a magnetic stir bar was charged with $(S)$-BINOL $(54 \mathrm{mg}, 0.19 \mathrm{mmol})$. Anhydrous toluene $(1 \mathrm{~mL})$ was added followed by $\mathrm{AlMe}_{3}(18.5 \mu \mathrm{L}$, $0.19 \mathrm{mmol}$ ) and the vial was capped with a Teflon-lined silicone septa. The resulting cloudy mixture was stirred for $0.5 \mathrm{~h}$ when 2-propanol $(50 \mu \mathrm{L}, 0.64 \mathrm{mmol})$ was added. The ensuing homogeneous reaction was allowed to stir at room temperature for an additional $0.5 \mathrm{~h}$, taken out of the drybox, and then heated to $60{ }^{\circ} \mathrm{C}$ in a Thermolyne Type 17600 DriBath block heater equipped with an aluminum heating block containing a $4 \times 3$ bank of [20 mm diameter $\times 50 \mathrm{~mm}$ depth] wells. A solution of the imine $(0.16 \mathrm{mmol})$ in toluene $(1 \mathrm{~mL})$ was then added via a gas-tight syringe and the resulting yellow solution was stirred for an additional $20 \mathrm{~h}$ at $60{ }^{\circ} \mathrm{C}$. The reaction was cooled to room temperature, diluted with $\mathrm{CH}_{2} \mathrm{Cl}_{2}(20 \mathrm{~mL})$, and washed with $\mathrm{H}_{2} \mathrm{O}(3 \times 50 \mathrm{~mL})$ and saturated aqueous $\mathrm{NaCl}(50 \mathrm{~mL})$. The organics were then dried over anhydrous $\mathrm{Na}_{2} \mathrm{SO}_{4}$, filtered over a Büchner funnel, and evaporated to dryness on a rotary evaporator. Crude reaction mixtures were then purified via chromatography on silica gel $(25 \mathrm{~cm} \times 2 \mathrm{~cm})$ with acetone $/ \mathrm{CH}_{2} \mathrm{Cl}_{2}$ eluants $(10 / 90-50 / 50)$. Stereochemical assignments of the products were made via comparison to literature precedents (vide infra).

$\boldsymbol{N}$-(1-Phenylethyl)-P,P-diphenylphosphinamide (11) ${ }^{3,4}$ A white solid (yield $=85 \%$, ee $=96 \%$ (Table 2, entry 1 )). ${ }^{1} \mathrm{H}$ NMR (400.64 MHz, $\left.\mathrm{CDCl}_{3}\right): \delta 1.50(\mathrm{~d}, J=6.8 \mathrm{~Hz}, 3 \mathrm{H}), 3.18(\mathrm{~b} \mathrm{~s}, 1 \mathrm{H}), 4.31(\mathrm{~m}, 1 \mathrm{H}), 7.16-7.42(\mathrm{~m}, 8 \mathrm{H}), 7.72-7.86(\mathrm{~m}, 4$ H). ${ }^{13} \mathrm{C}\left\{{ }^{1} \mathrm{H}\right\} \operatorname{NMR}(100.73 \mathrm{MHz}, \mathrm{CDCl} 3): \delta 26.2(\mathrm{~d}, J=3.0 \mathrm{~Hz}), 51.2,126.1,127.3,128.5-128.8(\mathrm{~m}), 131.9-132.2(\mathrm{~m})$, 132.6, 132.7, $145.2(\mathrm{~d}, J=6.8 \mathrm{~Hz}) .{ }^{31} \mathrm{P}\left\{{ }^{1} \mathrm{H}\right\}$ NMR $\left(\mathrm{CDCl}_{3}\right): \delta 23$. GC-MS (EI): Calculated for $\mathrm{C}_{21} \mathrm{H}_{22} \mathrm{NPO} 321.35$; Found $=321$. HPLC conditions: $10 \%{ }^{i} \mathrm{PrOH}$ in hexanes, flow rate $=0.5 \mathrm{~mL} / \mathrm{min}$, detector set at $254 \mathrm{~nm}$. Major isomer retention time $=13.5 \mathrm{~min}$, minor isomer retention time $=16.7 \mathrm{~min}$.

(a)

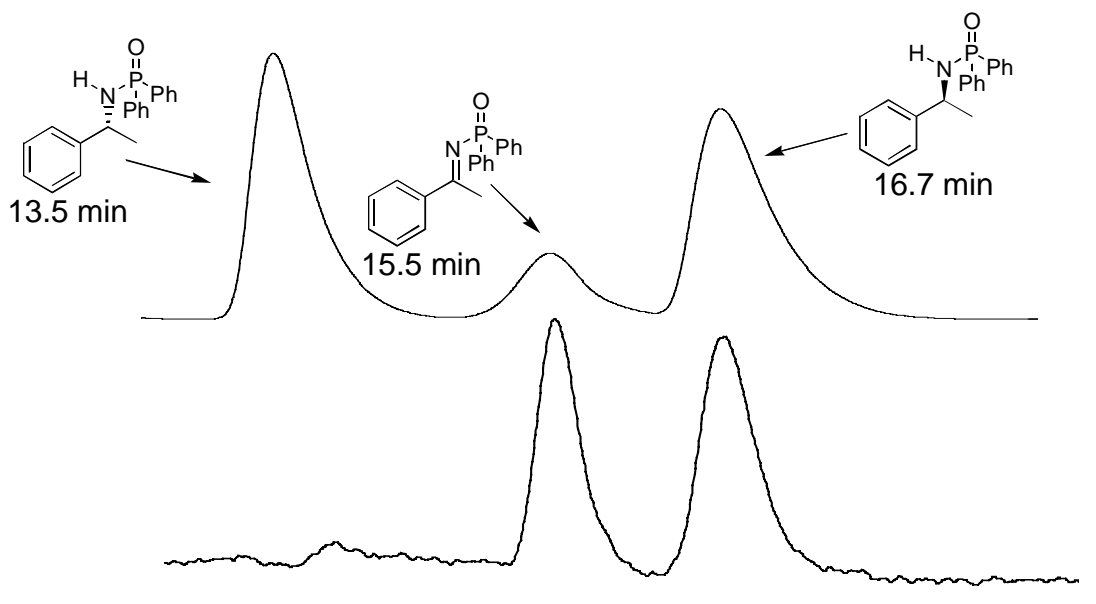

(b)

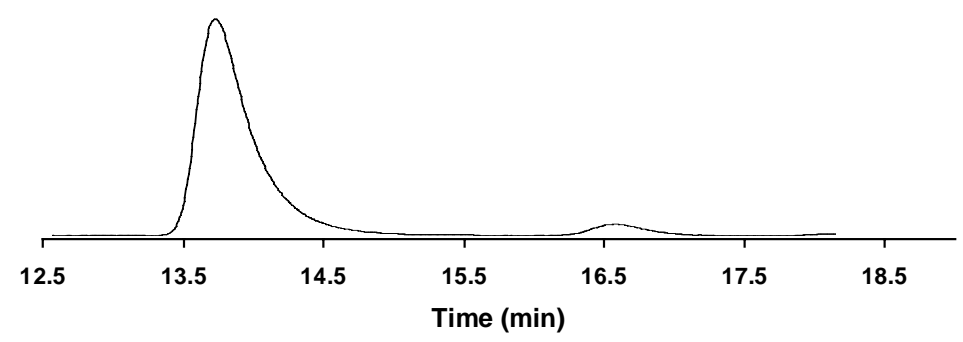

Figure S1. Chiral HPLC traces of 11: (a) crude reaction mixture from the $\mathrm{NaBH}_{4}$ reduction of 1, (b) crude reaction mixture from the $(R)-\mathrm{BINOL} / \mathrm{AlMe}_{3} /{ }^{i} \mathrm{PrOH}$ reduction of $\mathbf{1}$ (Table 3, entry 5), and (c) purified product from $(S)$ $\mathrm{BINOL} / \mathrm{AlMe}_{3} / \mathrm{PrOH}$ reduction of $\mathbf{1}$ (Table 2, entry 1). The large extinction coefficient of the imine starting material exaggerates its presence vs. that of the amine products in (a) and (b).

$\boldsymbol{N}$-(1-Phenylpropyl)-P,P-diphenylphosphinamide(12). ${ }^{3,4}$ A white solid (yield $=85 \%$, ee $=95 \%$ (Table 2 , entry 2 )). ${ }^{1} \mathrm{H}$ NMR (499.48 MHz, $\left.\mathrm{CDCl}_{3}\right): \delta 0.72(\mathrm{t}, J=7.5 \mathrm{~Hz}, 3 \mathrm{H}), 1.74-1.97(\mathrm{~m}, 2.5 \mathrm{H}) ; 3.18$ (b s, $\left.1 \mathrm{H}\right), 4.01-4.04(\mathrm{~m}, 1 \mathrm{H}), 7.06-$ $7.43(\mathrm{~m}, 10 \mathrm{H}) 7.67-7.81(\mathrm{~m}, 4 \mathrm{H}) .{ }^{13} \mathrm{C}\left\{{ }^{1} \mathrm{H}\right\}$ NMR $\left(100.74 \mathrm{MHz}, \mathrm{CDCl}_{3}\right): \delta 10.8,32.8,57.3,126.7,127.3,128.4,128.6-$ $128.7(\mathrm{~m}), 131.9-132.1(\mathrm{~m}), 132.8,132.9,143.7 .{ }^{31} \mathrm{P}\left\{{ }^{1} \mathrm{H}\right\}$ NMR $\left(\mathrm{CDCl}_{3}\right): \delta 23$. GC-MS (EI): Calculated for $\mathrm{C}_{21} \mathrm{H}_{22} \mathrm{NPO}$ 
335.38; Found $=334$. HPLC conditions: $10 \%{ }^{i} \mathrm{PrOH}$ in hexanes, flow rate $=0.5 \mathrm{~mL} / \mathrm{min}$, detector set at $254 \mathrm{~nm}$. Major isomer retention time $=12.9 \mathrm{~min}$, minor isomer retention time $=16.6 \mathrm{~min}$.

$\boldsymbol{N}$-(1-Phenylbutyl)-P,P-diphenylphosphinamide(13). ${ }^{5}$ An off-white solid (yield $=84 \%$, ee $=94 \%($ Table 2 , entry 3$)$ ). ${ }^{1} \mathrm{H}$ NMR (400.63 MHz, $\left.\mathrm{CDCl}_{3}\right): \delta 0.75(\mathrm{t}, J=7.2 \mathrm{~Hz}, 3 \mathrm{H}), 1.08-1.18(\mathrm{~m}, 2 \mathrm{H}), 1.72-1.86(\mathrm{~m}, 2 \mathrm{H}), 3.18(\mathrm{~b} \mathrm{~s}, 1 \mathrm{H}), 4.08(\mathrm{~m}, 1$ $\mathrm{H}), 7.06-7.42(\mathrm{~m}, 11 \mathrm{H}), 7.66-7.81(\mathrm{~m}, 4 \mathrm{H}) .{ }^{13} \mathrm{C}\left\{{ }^{1} \mathrm{H}\right\} \mathrm{NMR}\left(100.74 \mathrm{MHz}, \mathrm{CDCl}_{3}\right): \delta 14.0,19.6,42.2,55.9,126.6,127.3$, 128.4-128.7 (m), 131.9-132.1 (m), 132.6-132.9 (m), 143.1. ${ }^{31} \mathrm{P}\left\{{ }^{1} \mathrm{H}\right\}$ NMR $\left(\mathrm{CDCl}_{3}\right)$ : $\delta 23$. GC-MS (EI): Calculated for $\mathrm{C}_{22} \mathrm{H}_{24} \mathrm{NPO}=349.41$; Found $=348$. HPLC conditions: $10 \%{ }^{i} \mathrm{PrOH}$ in hexanes, flow rate $=1.0 \mathrm{~mL} / \mathrm{min}$, detector set at 254 $\mathrm{nm}$. Major isomer retention time $=4.9 \mathrm{~min}$, minor isomer retention time $=5.8 \mathrm{~min}$.

$\boldsymbol{N}$-(1-Phenyl-2-methylpropyl)-P,P-diphenylphosphinamide (14). ${ }^{6}$ A white solid (yield = 79\%, ee $=96 \%$ (Table 2 , entry 4)). ${ }^{1} \mathrm{H}$ NMR (400.63 MHz, $\left.\mathrm{CDCl}_{3}\right): \delta 0.78(\mathrm{~d}, J=7.2 \mathrm{~Hz}, 3 \mathrm{H}), 0.98(\mathrm{~d}, J=6.8 \mathrm{~Hz}, 3 \mathrm{H}), 1.97(\mathrm{~m}, 1 \mathrm{H}), 3.37(\mathrm{~m}, 1 \mathrm{H})$, $3.87(\mathrm{~m}, 1 \mathrm{H}), 7.03(\mathrm{~d}, J=6.8 \mathrm{~Hz}, 2 \mathrm{H}), 7.17-7.45(\mathrm{~m}, 9 \mathrm{H}), 7.60-7.91(\mathrm{~m}, 4 \mathrm{H}) .{ }^{13} \mathrm{C}\left\{{ }^{1} \mathrm{H}\right\} \mathrm{NMR}\left(100.73 \mathrm{MHz}, \mathrm{CDCl}_{3}\right): \delta$ 19.4, 35.8, 35.1, 61.5 (d, $J=1.5 \mathrm{~Hz}), 126.8,127.0,127.1,128.2-128.3(\mathrm{~m}), 128.6-128.7(\mathrm{~m}), 131.7-132.0(\mathrm{~m}), 132.7,132.9$, $143.1(\mathrm{~d}, J=3.8 \mathrm{~Hz}) .{ }^{31} \mathrm{P}\left\{{ }^{1} \mathrm{H}\right\} \mathrm{NMR}\left(\mathrm{CDCl}_{3}\right): \delta 23$. GC-MS (EI): Calculated for $\mathrm{C}_{22} \mathrm{H}_{24} \mathrm{NPO}=349.41$; Found $=348$. HPLC conditions: $10 \%{ }^{i} \mathrm{PrOH}$ in hexanes, flow rate $=1.0 \mathrm{~mL} / \mathrm{min}$, detector set at $254 \mathrm{~nm}$. Minor isomer retention time $=$ $6.4 \mathrm{~min}$, major isomer retention time $=7.8 \mathrm{~min}$.

$\boldsymbol{N}$-(1-(1-Napthyl)ethyl)-P,P-diphenylphosphinamide (15). ${ }^{7}$ An off-white solid (yield $=80 \%$, ee $=98 \%$ (Table 2 , entry 5)). ${ }^{1} \mathrm{H}$ NMR (400.63 MHz, $\left.\mathrm{CDCl}_{3}\right): \delta 1.63(\mathrm{~d}, J=6.4 \mathrm{~Hz}, 3 \mathrm{H}), 3.33(\mathrm{~b} \mathrm{~s}, 1 \mathrm{H}), 5.16(\mathrm{~m}, 1 \mathrm{H}), 7.19-7.44(\mathrm{~m}, 10 \mathrm{H}), 7.56$ $(\mathrm{d}, J=6.8 \mathrm{~Hz}, 1 \mathrm{H}), 7.62-7.87(\mathrm{~m}, 6 \mathrm{H}) .{ }^{13} \mathrm{C}\left\{{ }^{1} \mathrm{H}\right\} \mathrm{NMR}\left(100.73 \mathrm{MHz}, \mathrm{CDCl}_{3}\right): \delta 26.4,47.4,122.6,123.2,125.7,125.8$, 126.3, 128.0, 128.5-129.0 (m), 132.1-132.5 (m). ${ }^{31} \mathrm{P}\left\{{ }^{1} \mathrm{H}\right\}$ NMR $\left(\mathrm{CDCl}_{3}\right): \delta 24$. GC-MS (EI): Calculated for $\mathrm{C}_{24} \mathrm{H}_{22} \mathrm{NPO}=$ 371.41; Found $=371$. HPLC conditions: $10 \%{ }^{i} \mathrm{PrOH}$ in hexanes, flow rate $=1.0 \mathrm{~mL} / \mathrm{min}$, detector set at $254 \mathrm{~nm}$. Major isomer retention time $=8.8 \mathrm{~min}$, minor isomer retention time $=11.7 \mathrm{~min}$.

$\boldsymbol{N}$-(1-(2-Napthyl)ethyl)-P,P-diphenylphosphinamide (16). ${ }^{7}$ An off-white solid (yield = 75\%, ee = 96\% (Table 2 , entry 6)). ${ }^{1} \mathrm{H} \mathrm{NMR}\left(499.48 \mathrm{MHz}, \mathrm{CDCl}_{3}\right): \delta 1.69(\mathrm{~d}, J=6.5 \mathrm{~Hz}, 3 \mathrm{H}), 3.33(\mathrm{~b} \mathrm{~s}, 1 \mathrm{H}), 4.58(\mathrm{~b} \mathrm{~s}, 1 \mathrm{H}) 7.28-7.53(\mathrm{~m}, 9 \mathrm{H}), 7.67$ $(\mathrm{s}, 1 \mathrm{H}), 7.80-7.97(\mathrm{~m}, 7 \mathrm{H}) .{ }^{13} \mathrm{C}\left\{{ }^{1} \mathrm{H}\right\} \mathrm{NMR}\left(100.74 \mathrm{MHz}, \mathrm{CDCl}_{3}\right): \delta 26.1,51.4,124.5,124.7,126.0,126.4,127.8,128.1$, 128.6-128.8 (m), 132.0-132.3 (m), 132.6-132.8 (m), 135.5. ${ }^{31} \mathrm{P}\left\{{ }^{1} \mathrm{H}\right\}$ NMR $\left(\mathrm{CDCl}_{3}\right): \delta 23$. GC-MS (EI): Calculated for $\mathrm{C}_{24} \mathrm{H}_{22} \mathrm{NPO}=371.41$; Found $=371$. HPLC conditions: $10 \%{ }^{i} \mathrm{PrOH}$ in hexanes, flow rate $=1.0 \mathrm{~mL} / \mathrm{min}$, detector set at 254 $\mathrm{nm}$. Major isomer retention time $=10.2 \mathrm{~min}$, minor isomer retention time $=11.0 \mathrm{~min}$.

$\boldsymbol{N}$-[2-(E-4-Phenyl-but-3-enyl)]-P,P-diphenylphosphinamide (17). ${ }^{5}$ An off-white solid (yield $=84 \%$, ee $=94 \%($ Table 2 , entry 7)). ${ }^{1} \mathrm{H}$ NMR (499.48 MHz, $\left.\mathrm{CDCl}_{3}\right): \delta 1.37(\mathrm{~d}, J=6.0 \mathrm{~Hz}, 3 \mathrm{H}), 2.88(\mathrm{~b} \mathrm{~s}, 1 \mathrm{H}), 3.91(\mathrm{~m}, 1 \mathrm{H}), 6.17\left(\mathrm{dd}, J_{l}=16.0\right.$ $\left.\mathrm{Hz}, J_{2}=5.0 \mathrm{~Hz}, 1 \mathrm{H}\right), 6.37(\mathrm{~d}, J=16.0 \mathrm{~Hz}, 1 \mathrm{H}), 7.15-7.44(\mathrm{~m}, 11 \mathrm{H}), 7.87(\mathrm{~b} \mathrm{~m}, 4 \mathrm{H}) .{ }^{13} \mathrm{C}\left\{{ }^{1} \mathrm{H}\right\} \mathrm{NMR}(125.60 \mathrm{MHz}$, $\left.\mathrm{CDCl}_{3}\right): \delta 24.3,49.3,126.6,127.8,128.6-128.8(\mathrm{~m}), 129.9,132.0-132.2(\mathrm{~m}), 132.5-132.7(\mathrm{~m}), 133.6,136.9 .{ }^{31} \mathrm{P}\left\{{ }^{1} \mathrm{H}\right\}$ NMR $\left(\mathrm{CDCl}_{3}\right): \delta 23$. GC-MS (EI): Calculated for $\mathrm{C}_{22} \mathrm{H}_{22} \mathrm{NPO} 347.39$; Found $=347$. HPLC conditions: $10 \%{ }^{i} \mathrm{PrOH}$ in hexanes, flow rate $=1.0 \mathrm{~mL} / \mathrm{min}$, detector set at $254 \mathrm{~nm}$. Major isomer retention time $=7.2$ min, minor isomer retention time $=8.4 \mathrm{~min}$.

$\boldsymbol{N}$-[2-(4-Phenyl-but-3-ynyl)]-P,P-diphenylphosphinamide (18). ${ }^{8}$ A pale yellow solid $($ yield $=80 \%$, ee $=94 \%($ Table 2 , entry 8)). ${ }^{1} \mathrm{H}$ NMR (499.48 MHz, $\left.\mathrm{CDCl}_{3}\right): \delta 1.62(\mathrm{~d}, J=6.5 \mathrm{~Hz}, 3 \mathrm{H}), 3.26(\mathrm{~m} .1 \mathrm{H}), 4.29(\mathrm{~m}, 1 \mathrm{H}), 7.19-7.52(\mathrm{~m}, 11 \mathrm{H})$, 7.72-7.95 (m, $4 \mathrm{H}) .{ }^{31} \mathrm{P}\left\{{ }^{1} \mathrm{H}\right\}$ NMR $\left(\mathrm{CDCl}_{3}\right)$ : $\delta$ 24. GC-MS (EI): Calculated for $\mathrm{C}_{22} \mathrm{H}_{24} \mathrm{NPO}=345.37 ;$ Found = 345. HPLC conditions: $10 \%{ }^{i} \mathrm{PrOH}$ in hexanes, flow rate $=1.0 \mathrm{~mL} / \mathrm{min}$, detector set at $254 \mathrm{~nm}$. Major isomer retention time $=$ $7.2 \mathrm{~min}$, minor isomer retention time $=8.4 \mathrm{~min}$.

$\boldsymbol{N}$-(1-cyclohexylethyl)-P,P-diphenylphosphinamide (19)..$^{5}$ An off-white solid (yield = 84\%, ee = 94\% (Table 2, entry 9)). ${ }^{1} \mathrm{H}$ NMR $\left(400.63 \mathrm{MHz}, \mathrm{CDCl}_{3}\right): \delta 1.15-1.48(\mathrm{~m}, 6 \mathrm{H}), 1.52-1.95(\mathrm{~m}, 8 \mathrm{H}), 2.56(\mathrm{~m}, 1 \mathrm{H}), 3.01(\mathrm{~m}, 1 \mathrm{H}), 7.38-7.58(\mathrm{~m}, 6$ $\mathrm{H})$, 7.72-7.90 (m, $4 \mathrm{H}) .{ }^{13} \mathrm{C}\left\{{ }^{1} \mathrm{H}\right\} \mathrm{NMR}\left(125.60 \mathrm{MHz}, \mathrm{CDCl}_{3}\right): \delta 25.6,25.9,26.3,27.3,28.4,30.0,52.4,128.8-129(\mathrm{~m})$, 130.0, 131.2-131.3 (m), 132.0-132.2 (m), 132.3-132.4 (m), 135.2. ${ }^{31} \mathrm{P}\left\{{ }^{1} \mathrm{H}\right\} \mathrm{NMR}\left(\mathrm{CDCl}_{3}\right): \delta 27$. HPLC conditions: $10 \%$ ${ }^{i} \mathrm{PrOH}$ in hexanes, flow rate $=1.0 \mathrm{~mL} / \mathrm{min}$, detector set at $254 \mathrm{~nm}$. Major isomer retention time $=11.05 \mathrm{~min}$, minor isomer retention time $=11.8 \mathrm{~min}$.

$\boldsymbol{N}$-(3-octyl)-P,P-diphenylphosphinamide (20). A pale yellow oil (yield: $84 \%$; ee: $93 \%$ (Table 2, entry 10)). ${ }^{1} \mathrm{H}$ NMR $\left(400.63 \mathrm{MHz}, \mathrm{CDCl}_{3}\right): \delta 0.79-1.50(\mathrm{~m}, 16 \mathrm{H}), 2.64(\mathrm{~m}, 1 \mathrm{H}), 2.93(\mathrm{~m}, 1 \mathrm{H}), 7.20-7.51(\mathrm{~m}, 6 \mathrm{H}), 7.82-7.86(\mathrm{~m}, 4 \mathrm{H})$. ${ }^{13} \mathrm{C}\left\{{ }^{1} \mathrm{H}\right\}$ NMR $\left(100.74 \mathrm{MHz}, \mathrm{CDCl}_{3}\right): \delta$ 9.8, 14.2, 22.7, 25.3, $29.5(\mathrm{~d}, J=5.3 \mathrm{~Hz}), 31.9,36.3(\mathrm{~d}, J=5.3 \mathrm{~Hz}), 52.9,128.5-$ $129.0(\mathrm{~m}), 131,1,131.9,132.2-132.5(\mathrm{~m}) .{ }^{31} \mathrm{P}\left\{{ }^{1} \mathrm{H}\right\} \mathrm{NMR}\left(\mathrm{CDCl}_{3}\right): \delta 22$. GC-MS (EI): Calculated for $\mathrm{C}_{20} \mathrm{H}_{28} \mathrm{NPO} 329.42$; 
Found $=329$. HPLC conditions: $10 \%{ }^{i} \mathrm{PrOH}$ in hexanes, flow rate $=1.0 \mathrm{~mL} / \mathrm{min}$, detector set at $254 \mathrm{~nm}$. Major isomer retention time $=8.6 \mathrm{~min}$, minor isomer retention $=10.9 \mathrm{~min}$.

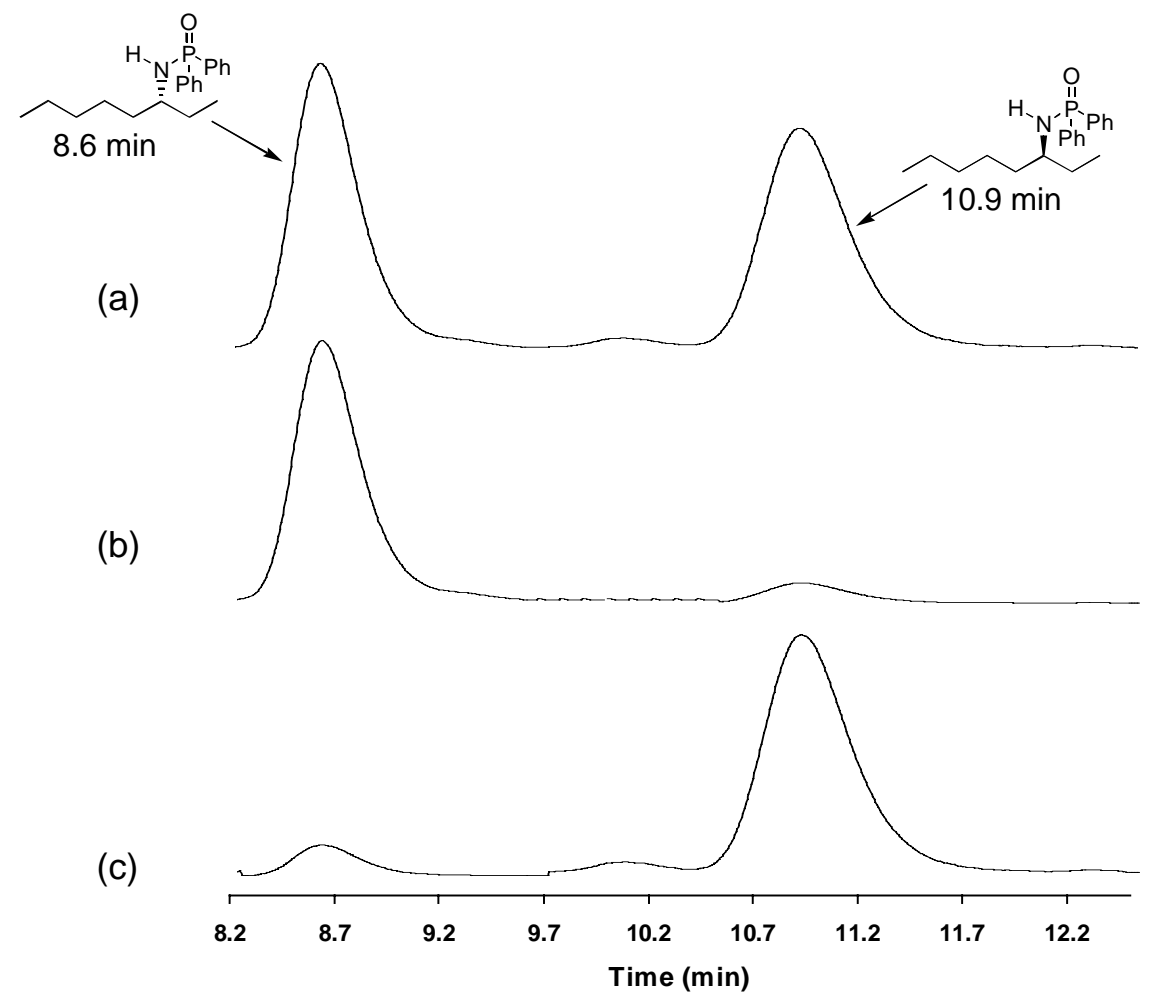

Figure S2. Chiral HPLC traces of 20: (a) crude reaction mixture from the $\mathrm{NaBH}_{4}$ reduction of 10, (b) purified product from $(S)$-BINOL/AlMe ${ }_{3} / \mathrm{PrOH}$ reduction of 10, and (c) crude reaction mixture from $(R)-\mathrm{BINOL} / \mathrm{AlMe}{ }_{3} / \mathrm{PrOH}$ reduction of $\mathbf{1 0}$.

Large scale MSPV reduction of 1 and BINOL recovery and recycling. A flame-dried 100-mL Schlenk flask equipped with a magnetic stir bar was charged with $(S)$-BINOL $(1.08 \mathrm{~g}, 3.8 \mathrm{mmol})$. The flask was evacuated and back-filled with nitrogen in triplicate, and anhydrous toluene $(33 \mathrm{~mL})$ was added via cannula under nitrogen. $\mathrm{AlMe}_{3}(2.0 \mathrm{M}$ solution in toluene, $1.9 \mathrm{~mL}, 3.8 \mathrm{mmol}$ ) was slowly added (over 1 minute) via a gas-tight syringe and the ensuing cloudy white mixture was stirred for $0.5 \mathrm{~h}$. Anhydrous 2-propanol $(1 \mathrm{~mL}, 12.8 \mathrm{mmol})$ was then added to the reaction via syringe to give a homogeneous, colorless solution, which was then allowed to stir for an additional $0.5 \mathrm{~h}$. The reaction vessel was heated to $60{ }^{\circ} \mathrm{C}$ via an oil bath and $1(1.0 \mathrm{~g}, 3.2 \mathrm{mmol})$ in anhydrous toluene $(5 \mathrm{~mL})$ was added to give a bright yellow solution. After $20 \mathrm{~h}$ stirring at $60{ }^{\circ} \mathrm{C}$, heating was stopped and $\mathrm{CH}_{2} \mathrm{Cl}_{2}(100 \mathrm{~mL})$ was added. The resulting mixture was extracted with aqueous $\mathrm{KOH}(5 \mathrm{wt} \%$ in water, $3 \times 50 \mathrm{~mL})$ and the aqueous layers were combined and set aside (vide infra). The organic layer was dried over anhydrous $\mathrm{Na}_{2} \mathrm{SO}_{4}$, and solvent was removed. Crude reaction product was purified by chromatography on silica (acetone/ $\left.\mathrm{CH}_{2} \mathrm{Cl}_{2}, 40 / 60\right)$ to give pure $(R)-\mathbf{1 1}^{3}$ as a white solid $(0.88 \mathrm{~g}, 86 \%$ yield, $96 \%$ ee) as well as $(S)$-BINOL $(0.1 \mathrm{~g})$.

The aqueous layer (vide supra) was acidified with $2 \mathrm{~N} \mathrm{HCl}(\sim 100 \mathrm{~mL})$ and extracted with $\mathrm{CH}_{2} \mathrm{Cl}_{2}(3 \times 50 \mathrm{~mL})$. The combined organic layers were dried over anhydrous $\mathrm{Na}_{2} \mathrm{SO}_{4}$ and filtered over a Buchner funnel. The filtrate was evaporated on a rotary evaporator and the resulting crude product was further dried in vacuo to leave $(S)$-BINOL as a white solid $(0.90 \mathrm{~g}, 83 \%$ recovery). Importantly, in the purification of the amine (vide supra) an additional crop of (S)-BINOL $(0.1 \mathrm{~g})$ was collected to give a total yield of $93 \%$ of recovered $(S)$-BINOL. This sample was used in a subsequent reduction of $\mathbf{1}$ following the procedure out line above to give the chiral product $\mathbf{1 1}$ in $95 \%$ ee $(90 \%$ conversion of $\mathbf{1})$.

Synthesis of deuterated amines. The general reaction set-up for the deuteration of $\mathbf{1}$ followed that of the general imine reduction strategy (vide supra) but with a larger amount of the imine (150 $\mathrm{mg}, 0.48 \mathrm{mmol})$. All other reagents were scaled accordingly with anhydrous 2-propan-2- $d$-ol being used in place of 2-propanol as the reducing agent. The crude reaction mixture was purified via column chromatography on silica gel $(25 \mathrm{~cm} \times 2 \mathrm{~cm})$ with acetone/ $\mathrm{CH}_{2} \mathrm{Cl}_{2}$ (40/60) eluant to yield $(R)-11-d$ as a white solid (116 mg, 75\% yield, $96 \%$ ee, Figure S3). ${ }^{1} \mathrm{H}$ NMR $\left(499.48 \mathrm{MHz}, \mathrm{CDCl}_{3}\right): \delta 1.58(\mathrm{~s}, 3 \mathrm{H}), 3.59(\mathrm{~b}$, 1H), 7.27-7.40 (m, 7H), 7.47-7.53 (m, 4H), 7.82-7.86 (m, 2H), 7.93-7.96 (m, $2 \mathrm{H}) .{ }^{13} \mathrm{C}\left\{{ }^{1} \mathrm{H}\right\} \mathrm{NMR}(125.60 \mathrm{MHz}, \mathrm{CDCl} 3)$ : 
$\delta 26.1(\mathrm{~d}, J=2.5 \mathrm{~Hz}), 51.1(\mathrm{t}, J=19.6 \mathrm{~Hz}), 126.2,127.4,128.7-128.8(\mathrm{~m}), 132.1-132.3(\mathrm{~m}), 132.7,132.8,145.2(\mathrm{~d}, J=7.2$ $\mathrm{Hz}) .{ }^{31} \mathrm{P}\left\{{ }^{1} \mathrm{H}\right\}$ NMR $\left(\mathrm{CDCl}_{3}\right): \delta 24(\mathrm{~s}) .{ }^{2} \mathrm{H}\left\{{ }^{1} \mathrm{H}\right\}$ NMR $\left(\mathrm{CH}_{2} \mathrm{Cl}_{2}\right): \delta 4.30(\mathrm{~s})$. GC-MS (EI): Calculated for $\mathrm{C}_{20} \mathrm{H}_{19} \mathrm{DNPO}=$ 322.36; Found $=322$. HPLC conditions: $10 \%{ }^{i} \mathrm{PrOH}$ in hexanes, flow rate $=0.5 \mathrm{~mL} / \mathrm{min}$, detector set at $254 \mathrm{~nm}$. Major isomer retention time $=13.8 \mathrm{~min}$, minor isomer retention time $=17.2 \mathrm{~min}$.

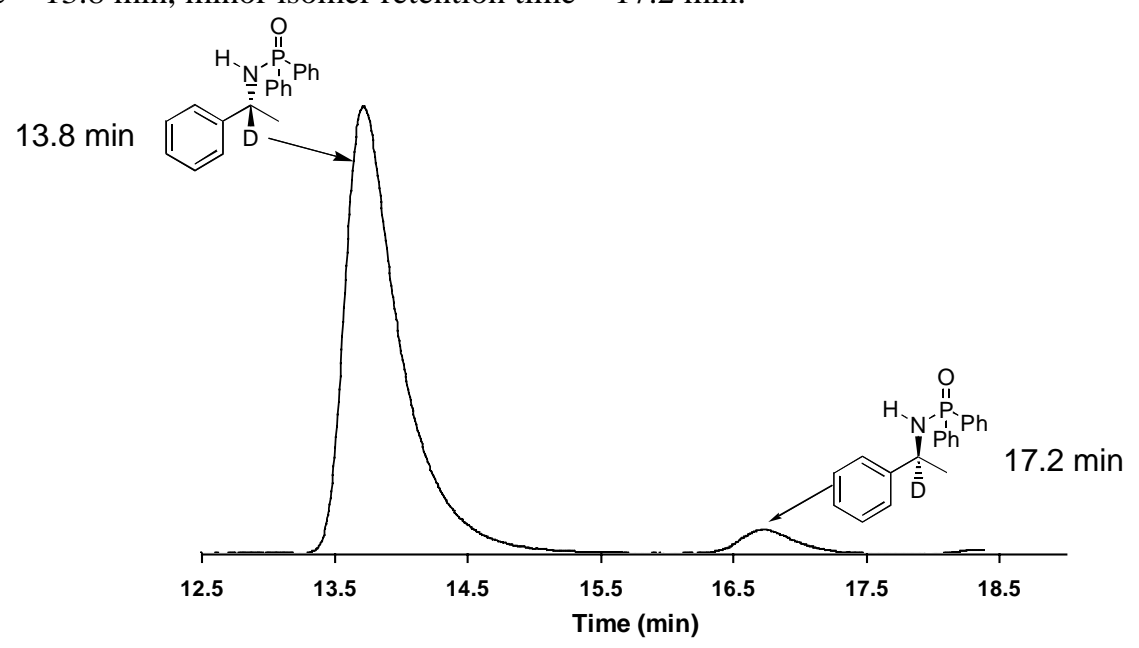

Figure S3. Chiral HPLC traces of the purified product from the reduction of 1 with $(S)$-BINOL/AlMe $3 / 2-$ propan-2- $d$-ol.

The product $(S)$-20- $d$ could be synthesized in 93\% ee following an analogous manner implementing $(R)$ BINOL/AlMe ${ }_{3}$ in the reduction of 10 (Figure S4). ${ }^{1} \mathrm{H}$ NMR $\left(400.64 \mathrm{MHz}, \mathrm{CDCl}_{3}\right): \delta 0.75-1.48(\mathrm{~m}, 16 \mathrm{H}), 2.45(\mathrm{~m}, 1 \mathrm{H})$, 7.23-7.51 (m, $6 \mathrm{H}), 7.80-7.86(\mathrm{~m}, 4 \mathrm{H}) .{ }^{13} \mathrm{C}\left\{{ }^{1} \mathrm{H}\right\}$ NMR (100.74 MHz, $\left.\mathrm{CDCl}_{3}\right): \delta$ 9.8, 14.2, 22.7, 25.3, 29.8, 31.9, 36.1, 51.7 128.0-129.2 (m), 131.3-132.5 (m). ${ }^{31} \mathrm{P}\left\{{ }^{1} \mathrm{H}\right\} \mathrm{NMR}\left(\mathrm{CDCl}_{3}\right): \delta 17 \mathrm{ppm} .{ }^{2} \mathrm{H}\left\{{ }^{1} \mathrm{H}\right\} \mathrm{NMR}\left(\mathrm{CH}_{2} \mathrm{Cl}_{2}\right): \delta 3.47(\mathrm{~s})$. GC-MS (EI): Calculated for $\mathrm{C}_{20} \mathrm{H}_{19} \mathrm{DNPO}=330.42$; Found $=330$. HPLC conditions: $10 \%{ }^{i} \mathrm{PrOH}$ in hexanes, flow rate $=1.0$ $\mathrm{mL} / \mathrm{min}$, detector set at $254 \mathrm{~nm}$.

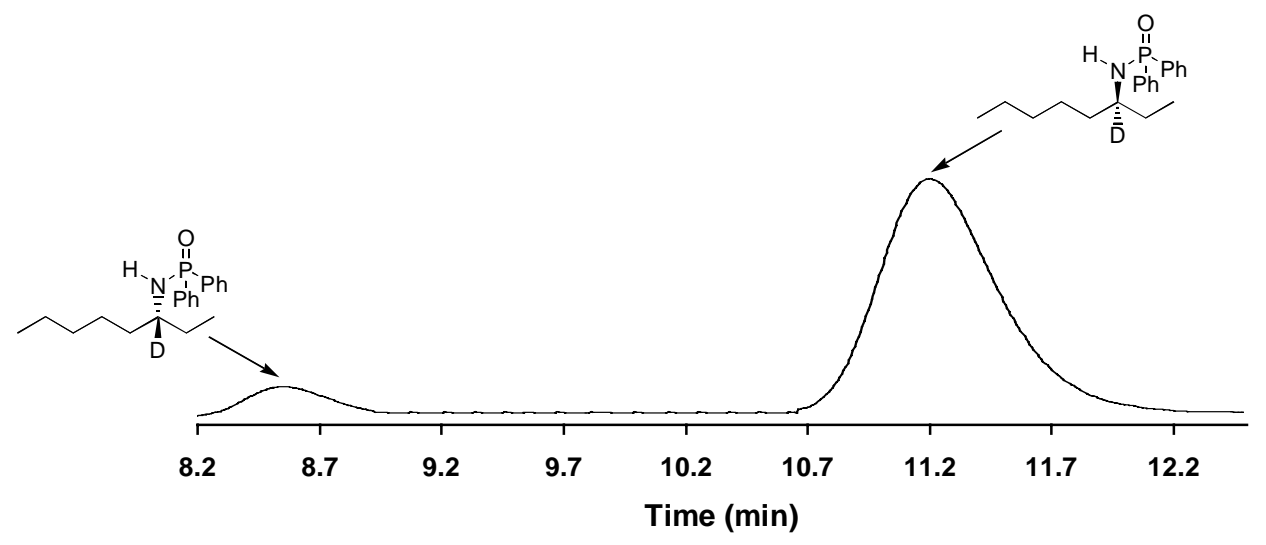

Figure S4. Chiral HPLC traces of the purified product from the reduction of $\mathbf{1 0}$ with $(R)$-BINOL/AlMe $3 / 2$-propan-2- $d$-ol.

Procedure for ${ }^{27} \mathrm{Al}$ NMR experiment. In a drybox, to a 4-mL vial equipped with a magnetic stir bar was added $(S)$ BINOL (96 mg, $0.32 \mathrm{mmol}), \mathrm{C}_{6} \mathrm{D}_{6}(1 \mathrm{~mL})$, and $\mathrm{AlMe}_{3}(33 \mu \mathrm{L}, 0.32 \mathrm{mmol}$ via a gas-tight syringe). The ensuing cloudy mixture was stirred for $0.5 \mathrm{~h}$ when 2-propanol $(50 \mu \mathrm{L}, 0.32 \mathrm{mmol})$ was added. The resulting homogeneous reaction was stirred for an additional $0.5 \mathrm{~h}$ when compound 1 was added. The reaction instantaneously turned bright yellow and was stirred for $1 \mathrm{~h}$ at room temperature after which it was transferred to a gas-tight NMR tube and directly analyzed by NMR spectroscopy. ${ }^{27} \mathrm{Al}$ NMR: $\delta 45$ (b). ${ }^{31} \mathrm{P}\left\{{ }^{1} \mathrm{H}\right\}$ NMR: $\delta 22$ (s). The parent imine exhibits a single resonance $\left({ }^{31} \mathrm{P}\left\{{ }^{1} \mathrm{H}\right\}\right.$ NMR $\left.\left(\mathrm{C}_{6} \mathrm{D}_{6}\right): \delta 17\right)$ in its ${ }^{31} \mathrm{P}$ NMR spectrum. 


\section{References}

1) Pangborn, A. B.; Giardello, M. A.; Grubbs, R. H.; Rosen, R. K.; Timmers, F. J. Organometallics 1996, 15, 1518-1520.

2) Krzyzanowska, B.; Stec, W. J. Synthesis 1982, 270-273.

3) Guijarro, D.; Pinho, P.; Andersson, P. G. J. Org. Chem. 1998, 63, 2530-2535.

4) Andersson, P. G.; Guijarro, D.; Tanner, D. J. Org. Chem. 1997, 62, 7364-7375.

5) Nolin, K. A.; Ahn, R. W.; Toste, F. D. J. Am. Chem. Soc. 2005, 127, 12462-12463.

6) Buchowiecki, W.; Grosman-Zjawiona, Z.; Zjawiony, Z. Tetrahedron Lett. 1985, 26, 1245-1248.

7) Hutchins, R. O.; Abdel-Magid, A.; Stercho, Y. P.; Wambsgans, A. J. Org. Chem. 1987, 52, 702-704.

8) Wipf, P.; Stephenson, C. R. J.; Walczak, M. A. A. Org. Lett. 2004, 6, 3009-3012. 